\title{
Groupware for Developing Critical Discussion Skills
}

\author{
Dan Suthers and Arlene Weiner \\ Learning Research and Development Center, University of Pittsburgh
}

\begin{abstract}
Increasingly, social and collaborative processes are seen as central to the development of thinking skills, including the skills of scientific thinking. With colleagues we have developed a software system (Belvedere) to support high-school science students in engaging in collaborative reasoning and argumentation. We discuss how Belvedere is designed to support students' collaborative processes, describe our formative evaluation studies, and provide examples of student sessions. Reflecting on students' inter-actions with Belvedere and each other, we then discuss the competencies and limitations of students in developing scientific argumentation and reasoning, as distinguished from and supported by their practice of everyday argumentation..
\end{abstract}

Keywords - interface design, groupware, scientific argumentation, collaborative learning.

\section{Introduction}

Scientific knowledge has an increasing dynamism in two senses: it changes and increases extremely rapidly, and it is thrust from the lab into the wider world and the public forum almost as rapidly. These trends place increasing demands on secondary school science education. Besides knowing key facts and concepts and particular procedures, it is important for today's students to understand the processes by which the claims of science are generated, evaluated, and revised. In addition to efforts being made to restructure what is taught, there are grounds to change how it is taught. In particular, some researchers and educators have recommended collaborative discussion for learning science concepts and reasoning $[10,11,12,14,15,20]$.
Towards this end, we are experimenting with a software environment called "Belvedere" for supporting students learning to engage in critical discussion of competing scientific theories. In this paper we discuss some issues in using computers to support collaborative dialogues. We draw on our work with students using Belvedere to illustrate both student competencies that facilitate their practice of collaborative discussion of theories and affordances to facilitate their progress in such practice.

\section{An Experimental Argumentation Environment}

Belvedere is an environment designed to support the practice of critical discussion in a range of collaborative paradigms. It can be used jointly by students who are physically close together to work simultaneously on a shared argument; by students to work on shared arguments at different times; and, a use we have not been testing, by students working simultaneously but remote from each other. Superficially, Belvedere is networked groupware for constructing representations of the logical and rhetorical relations within a debate. The interface appears similar to drawing program (Figure 1), but using it feels more like assembling components into desired configurations. However, the utility of Belvedere's representations lies in the stimulation and coordination of discourse that takes place external to the representations themselves, as much as in their modeling value. Belvedere differs from other computer systems for supporting argumentation $[6,9,16]$ because it is designed to support students who may not have general skills of constructing arguments or specific knowledge of a domain. Thus the design of Belvedere addresses the cognitive and motivational requirements and limitations of these unpracticed 


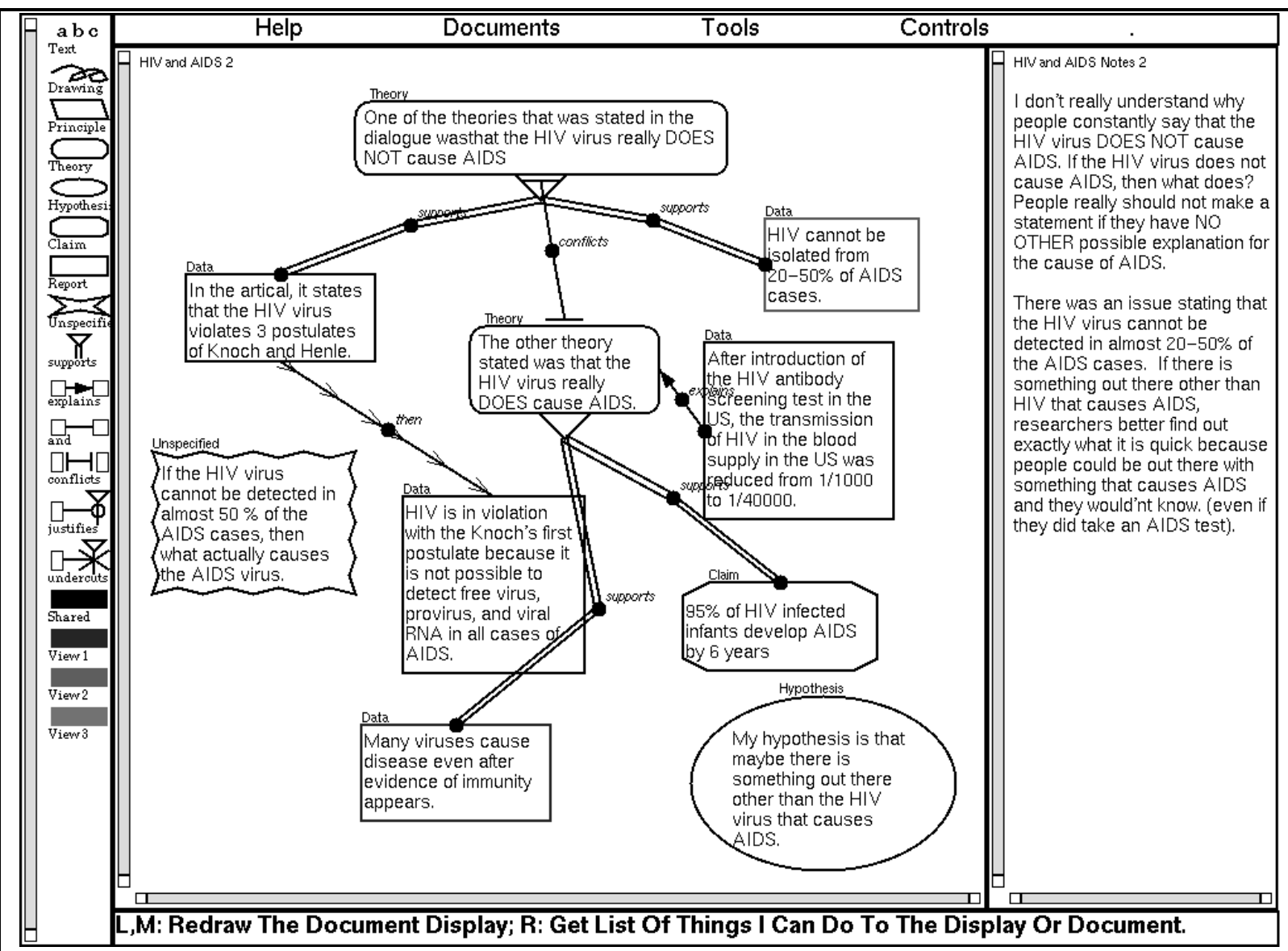

Figure 1: Diagram by Students on HIV and AIDS Issue.

beginners (discussed below). Belvedere differs from other systems for supporting students' discourse [13, 14] primarily in being designed to scaffold discourse about scientific theories and evidence.

\subsection{Supporting Participation in Critical Discussion}

After motivating the design in terms of how software tool can facilitate students' participation in critical discussion of science issues, we give some detailed comments on the design.

Reifying the Abstract Relationships Implicit in Scientific Theories and Arguments. Part of expertise in a domain is the ability to represent situations in terms of deep features or structures that are useful for solving problems in the domain $[4,5,7]$. Belvedere provides students with concrete forms for representing the abstract components and relationships of theories and related arguments. Ideas and relationships are represented as objects that can be pointed to, linked to other objects, and discussed.

Motivating by Shared Activity. Small-group work and the production of documents that will be used by others can provide peer motivation and a sense of authentic activity that teacher- and evaluation-centered work may not provide $[2,14,15,20]$. To support small group collaboration while allowing each student equal opportunity for input, Belvedere is networked so that students can work concurrently on the same diagram. The reified arguments enable students to jointly focus on and discuss the same claim, simultaneously and independently address different points, and switch between joint and independent work without losing track of the discussion.

Directing Attention to the Important Issues in a Complex Debate. Argumentation diagrams can help students identify ways in which further contributions can be made to the argument $[16,17]$. As the students build an argument, they can request advice. The automated advisor helps students focus on particular aspects of a complex issue by suggesting ways in which their diagrams can be extended or improved [18]. The advisor highlights objects in the diagram as possibly needing attention and offers hints based on principles such as consistency, empirical support, maximizing a theory's coverage, and considering alternative theories. 
Providing Knowledge Resources. Belvedere provides facilities for authoring on-line knowledge resources that can be accessed and copied by students. Using these facilities we have authored modest collections of information in several scientific fields. Recently, Arthur Nunes has extended Belvedere to serve as a World Wide Web browser, enabling authors to use existing HTML tools.

\subsection{Selected Design Details}

Belvedere is implemented in the Common Lisp Interface Manager and ISI's LOOM, and runs in both Lucid Common Lisp on Decstations and Macintosh Common Lisp. We describe several aspects of the implementation briefly here.

The Graphical Language. The graphical forms we provide for argument representation are loosely based on the analysis developed by the philosopher Stephen Toulmin [19]. The representations are specialized for scientific argumentation by providing shapes for different types and components of arguments, negative as well as positive links, and enclosure and multiple linkages to accommodate complex arguments (see figure 1). A special "undefined" shape is provided for use when the epistemological status of a statement is not obvious or agreed upon. Different kinds of links between shapes represent different logical and rhetorical relations between the different statements. Colors are available to distinguish different viewpoints such as different theories or the contribution of each participant. Line thickness can be changed to express importance. Students can type text in to their diagrams or copy and paste excerpts from on-line or World-Wide Web documents. A primitive freehand drawing tool is also provided to enable participants to extend the representational repertory. Labels can be displayed for either shapes or links or both, and students can optionally change the labels and the shapes.

The Display. Belvedere is a symbol system for the expression of logical and rhetorical relations within a debate. We wanted participants to focus cognitive effort on the debate rather than on learning to use the program. Thus we made the interface look familiar by using command and icon layouts similar to those of typical drawing programs. We help maintain the students' focus on their understanding of the theories and controversies, rather than on every graphical detail of their diagrams, by automating some of the secondary aspects of the work. For example, graphical shapes are created with a default size, and resize themselves to fit their contents. When an object is moved, its links follow it to retain the logical connection. Other tools such as the automated advisor provide further relevant functionality not available in drawing programs.
Management of Concurrent Activity. In our initial studies with students sharing a single machine, some students appeared frustrated when limited to mouse operation while a partner dominated the input by typing on the keyboard. To avoid censorship based on who owns the input devices, we enable separate machines to display a shared document. Thus students can work concurrently on a shared diagram. Additional functionalities were required to manage this so as to minimize unnecessary redisplay overhead and maximize the students' focus on cognitive tasks. They should not be distracted by a constantly changing screen while they are thinking. Also, participants must not be able to operate on the same object simultaneously. We therefore "lock" an object as soon as a participant starts to use it. When it is locked, other users cannot modify it. Nor do they see the object changing: this would be annoying to someone pursuing their own thoughts, and require excess redisplay overhead. When the participant is finished editing and releases the lock, a redisplay interrupt is sent to other participants' applications. This interrupt is delayed by any applications in which another participant is editing, to avoid unexpected change of the context of their work.

\section{Formative Evaluations}

We discuss our formative evaluations to convey our experiences with both the advantages and dangers of collaborative learning processes.

Initial formative evaluation included several task scenarios tried with 8 paid subjects, ages 12 to 15 , working alone or in pairs, in 17 different 2-hour sessions in our laboratory. We ran three sets of laboratory studies, starting with an early prototype.

In the first set we were primarily concerned with the usability of the interface and the suitability of the diagrammatic representation. Individual students were briefly introduced to the representation and were acquainted with the interface. Then they were given a text presenting a scientific theory about the origins of mountains and asked to use Belvedere to show the ideas in the text. Considerable revision of interface details resulted from these sessions.

In the second set of studies, students from the previous studies worked in pairs using one computer. Each pair was asked to attempt to resolve an apparent anomaly for the Darwinian theory of adaptive radiation. (According to this theory, the unique species of the Galapagos islands separated on the islands in response to opportunities there. Molecular-biological dating indicates that the marine and land species of iguanas on the Galapagos diverged from a common 
ancestor about 12 million years ago, but radioisotope dating of rock gives an age of only 3 million years for the islands [3].) The initial conflict was presented in both textual and graphical forms. Students could call up texts from a modest database of small pieces of information, most of which was relevant to the problem, although not always in obvious ways. We were encouraged by the amount of discussion we saw in some pairs. However, in some cases the student who obtained the keyboard dominated and censored the other student's proposals for the diagram.

These observations motivated redesign of Belvedere for the third set of studies, identical to the second except that the students worked on individual computers networked together and located side by side (close enough to see and point to each other's displays). The monitors displayed a shared drawing space and a display in which each of the students could bring up texts from the database. This networked configuration was tested with two pairs of students in the laboratory, and subsequently with many more in the schools.

At this point we were satisfied that the prototype environment had potential value for students, and we began studying how 10th grade students and their science teachers might learn and use Belvedere in a classroom. The school is an urban school in a low-SES neighborhood. Its students have access to a computer lab and thus most of the students were familiar with a low-end Macintosh and mouse. One activity of the teachers and students during the time we observed them was preparation for a local science fair. This gave us the opportunity to observe some students' presentations of library research and later of project findings. Our informal impressions were consistent with others' ethnographic observation that discussion was teacher-initiated and teacher-centered, and that students' talk was social or task-related rather than conceptual [10].

One of the teachers suggested that we use a debate she had recently seen on an electronic discussion group: whether HIV is the cause of AIDS. Using a published summary of the scientific sources of the debate $[1,8]$, we developed a small database for theories of the cause of AIDS. Eight sessions were conducted in which two to three students worked on shared documents from their own machines, but sitting close enough to talk and see each other's displays. Figure 1 shows the argument diagram produced by one pair of students after two half-hour sessions (which included the time needed to learn to use the Belvedere system for the first time). Note that it includes not only the argument against causation but also some beginnings of rebuttal to it.

\section{Students' Competencies and Needs}

Most of the students in the collaborative studies created nodes, filled them with text (either typed in or copied) and linked them. Only one student completely misunderstood the representation, attempting to use Belvedere as a pictorial rather than a symbolic drawing program. The students in the classroom seemed to have clear opinions on the AIDS issue. Many students in both the lab and the classroom studies brought in knowledge and personal experiences from outside the textual materials provided to them. Most students were able to incorporate several points of the debate into their diagrams, drawing on the printed texts and/or the on-line information provided them, even in the short span of a class period. When prompted, most students were able to discuss what they thought about various aspects of the two positions, even if they were unable or unwilling to incorporate their thoughts into a Belvedere pane. Apart from collaborative processes to be discussed, difficulties arose primarily from problems with the software, limited screen size, and lack of clarity concerning the task posed to them.

\subsection{Generating Multiple Hypotheses}

One of the potential strengths of collaborative work is that alternate hypotheses and multiple perspectives may be considered. Consistent with this claim, subjects in dyads or triads usually generated several hypotheses (in contrast to observed science-fair monological presentations). For example, one dyad generated these hypotheses for the Galapagos problem: (1) the dating of the species' divergence may be wrong; (2) the islands may have been dated using younger surface rocks formed during volcanism subsequent to the initial formation of the island; (3) "the Galapagos islands may have moved near to another land mass where the iguanas were found" (subsequently this student asked about tectonic plate movement); and (4) when the Galapagos were forming, another island near them had the two species on it already. In an HIV and AIDS session (not pictured), discussion was focusing on discrepancies between the time during which HIV is most active, the onset of immunity, and the onset of AIDS symptoms. One student suggested that two disease processes may be involved; another suggested that HIV may not be the cause of AIDS but enables contracting AIDS. Typically, more verbal proposals were made than were entered into the diagram.

\subsection{Peer Coaching}

Students also complemented each other with background knowledge, or by spontaneously explaining the use of the interface or the meaning of the terms to each other. For example, a student 
$[3: 38]$

Mo: Do you want to put anything in, how ... we could always put something ... like, land animals, it says they could, like, travel on rafts of vegetation or on logs.... Do you want to put that in, because that's a way that they could've both migrated to the islands.

Emin: OK.

[3:57]

Emin: If there were no marine iguanas anywhere in the world except for the Galapagos, wouldn't that mean that they had developed from the

land iguanas, and not from any other ... the land-?

Mo: Um, well, they could've, but I think what, uhh-

Emin: Or they would've found, they might've found a climate, like, any other climate-

Mo: It says these two species separated about 12 million years ago, but maybe, like, and the islands weren't around then, so probably what happened is the marine iguanas died out by then everywhere else. Like, not by then, but by the time they got to the Galapagos islands they died off everywhere else, maybe. Does that make sense?

Emin: Yeah. So, do you want to put this in there, and then ... type what you just said? That they probably died out by then.

[4:01]

Mo: OK, now let me see what this says ... [read text of document indicating that marine iguanas do not swim for long distances.] ... But that wasn't a very long distance so that doesn't count. Because Ecuador to-

Emin: So the land iguanas probably developed from the marine iguanas, because-

Mo: Well, yeah, but they also, see, that's why we put that, they can go on the rafts and-

Emin: How did they get there in the first place? On the vegetation or whatever?

Mo: Yeah, the rafts or on logs.

Emin: Hey, look; the marine iguanas don't breathe under water.

Mo: They don't breathe under water?

Emin: They hold their breath.

Mo: Well, that's OK. That doesn't matter.

Emin: Well, it shows that they probably evolved from the land.

Mo: No, because they can always, um, come up if they're swimming under water ... [examining map of location of the Galapagos relative to South America] 600 miles; well ... that's still not too far ... It looks close on the map.

[4:28]

Emin [upon opening document that states that no marine iguana fossils have been found anywhere in the world other than the Galapagos]:

Uh-oh. [Emin points out contents to Mo; Mo groans]

[4:52]

Emin: Look; the second one [points in R-pane], it kind of goes with these two top ones [points to \#4 and 5], that they developed from ordinary land ones, because no fossils had been found, and no marine ones are anywhere else.

Mo: Umm ... That could be connected to either this one, right here [mouses over \#c], or it could be connected to these two [mouses over \#4 and 5], I think. I think either way you could connect it. Because you could say that because they separated here [mousing over \#c], they could've, umm, the marine could have died out, the marine iguanas, and then they could have, like, been reborn almost.

Emin: Why would they die out?

Mo: I don't know. [laughs] Somehow they could have died...

Emin: Died out on the islands? Because there were no fossils found anywhere else in the world.

Mo: Yeah ... Well, they could've died out when those islands weren't around then. So they probably - They could've just died out wherever they

were, and then, umm, the land iguanas, when they got to the islands, they, then in 2-3 million years, the marine iguanas developed from them.

Emin: But, you're saying that they died- that they were living someplace else and they died, and the land iguanas went to the Galapagos

islands, and new marine iguanas were born again?

Mo: Because there's no proof that says that the original marine iguanas are the same as the ones that are right now. But I don't know-

Emin: Yeah, I see what you mean.

Emin: [A few minutes later] OK, but if they migrated separately, how did the marine ones get there in the first place, if no fossils have been found elsewhere? They had to start out from someplace.

Mo: Oh. Well maybe they just haven't found any yet. [Emin laughs] The fossils are always beneath the water; maybe they just didn't, I'm sure

it's hard to find fossils underneath the water.

Emin: They might've died on land. [laughs]

[5:07]

Emin: Well, I think the answer is that just the land iguanas came from South America, by floating vegetation or whatever, and a subspecies developed off them which were the marine iguanas, since their fossils weren't found anywhere else.

Mo: I sort of agree with these two [points at bottom two ovals in R-pane], that the two kinds of iguanas separated on islands that are now worn down that are closer to South America, and then they both could have migrated to the Galapagos Islands, because marine animals- marine iguanas aren't found anywhere else, that would, like, explain that.

Emin: I'd like information on whether or not those islands, the worn-down islands ... if there were fossils and stuff-

Mo: Yeah, more on if, uhh-

Emin: ... other possibilities.

Mo: Where they've searched for fossils, too.

Table 1: Selections from Dialogue Between "Emin" and "Mo," Galapagos Iguana Anomaly

wondered what shape to use for the violation of the 2 postulates of Koch and Henle in Figure 1: "This would be like data, I think so, or a claim, would it be a claim or data?" Her partner replied: "Claim. Cause they don't have no real hard evidence, go ahead, claim. I mean who cares, who cares what they say? Claim." However, a peer's argument can lack authority: she chose "data."

\subsection{Conceptual Change}

When conflicting hypotheses were proposed, some groups engaged in a dialectic tension between challenges and resistance to change that provides a potential microcosm of scientific debate. For example, consider the dialogue of a Galapagos-problem dyad, selected portions of which are reproduced in Table 1. (The corresponding diagram is shown in Figure 2. The students chose pseudonyms.) Initially (3:38 of Table 1) Mo proposes that the two species came separately to the islands by floating or swimming. Emin then begins to encounter evidence that leads her to question this idea (3:57, 4:01 and 4:28), but Mo rather consistently dismisses it in order to maintain her reigning 


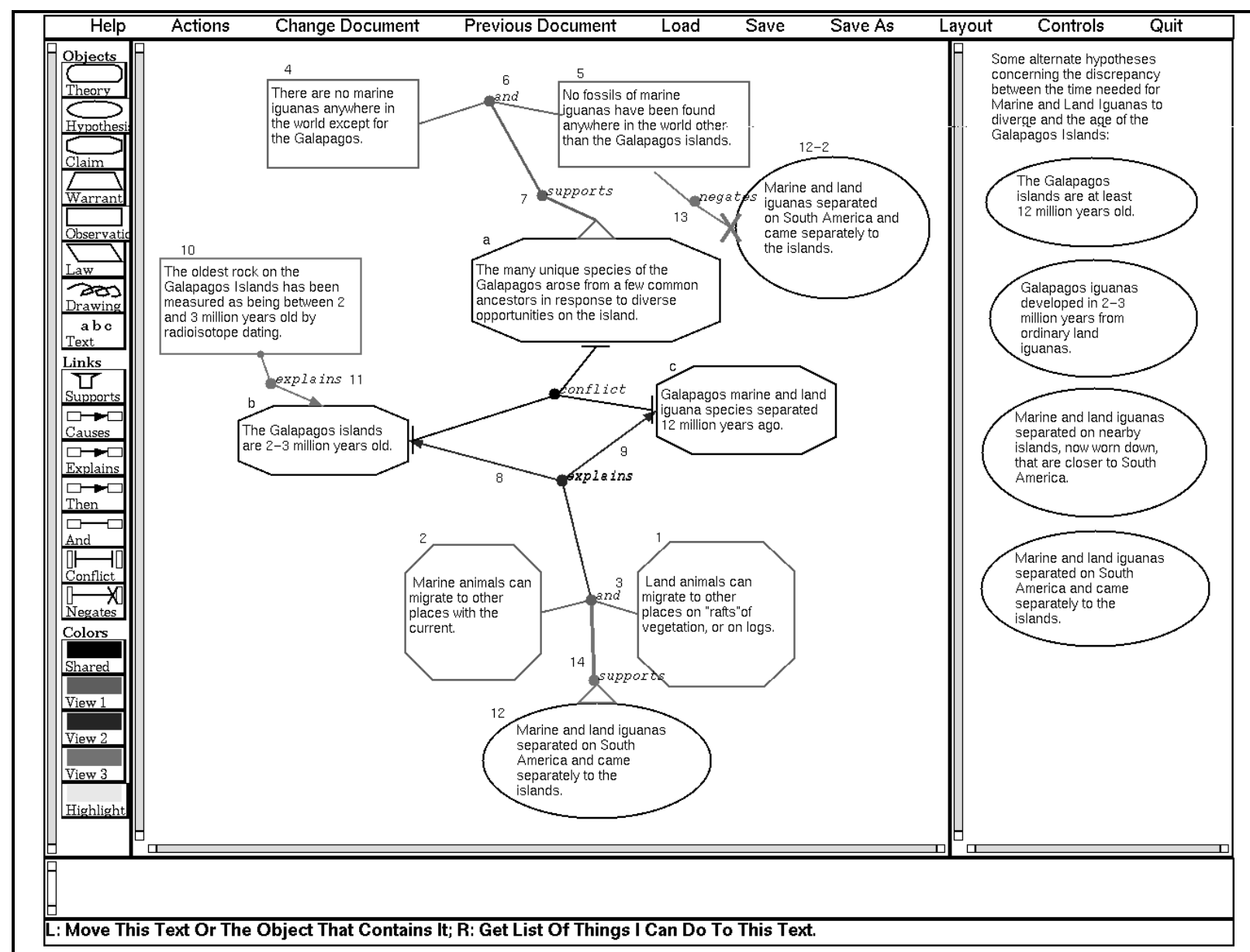

This session was conducted in an earlier version of Belvedere. The alternate hypotheses pictured were presented to them near the end of the session. Typically, only a portion of ideas and relationships discussed are captured in student diagrams (compare figure. to Table 1).

Figure 2: Diagram by "Emin" and "Mo," Galapagos Iguana Anomaly.

hypothesis. Later, we presented them with four alternate hypotheses to consider. Subsequently (4:52), Emin gently challenges Mo's conception, apparently on the basis of parsimony. By the end of the session (5:07), Mo gives up her former hypothesis and chooses one of the new ones. (The "worn-down islands" that she refers to are nearby sea-mounts, dated to be older than the Galapagos and bearing evidence of having once been above sea level.) While we cannot confidently identify the cause of Mo's change, we believe that dialogues such as this, with appropriate scaffolding and reflective follow-up, can provide the basis for personal experience of scientific dialectics.

\subsection{Social Impediments to Learning}

However, social processes do not always guarantee positive results. For example, in the first Galapagosproblem dyad, there was a subdued management struggle as one student wished to pursue a single line of argument and the other suggested that they should make a lot of hypotheses first. One student became frustrated when she suggested "maybe the dating is wrong," and her partner (in control of the keyboard) replied, "It can't be wrong, it's DNA." Even after we provided subsequent dyads with independent input devices, censoring persisted, albeit to a lesser degree. For example, Emin, was not always able to withstand Mo's confidence and be heard. At one point Emin justifiably challenges Mo, only to agree (in an unconvincing tone) a moment later, "I see what you mean" ([4:52] in Table 1). Thus, in an effort to be politely consensual throughout, a cooperative pair or group may fail to make the most of individual knowledge and judgment.

After persistently challenging Mo's conception that the marine and land iguanas separated elsewhere, Emin concluded that the species separated on the Galapagos ([5:07]), apparently forgetting the dating discrepancy posed at the outset. Her challenges to Mo indicate that she did not lack the ability to appreciate and address 
conflicts. Thus, even competent students may need help in keeping track of issues in a complex debate.

Finally, scientific argumentation is a specialized skill, and so requires an apprenticeship to practices not found in one's peer group. For example, look at the right-hand panel in Figure 1. It is common in everyday argumentation to ask critics to be constructive. As shown in the figure, one participant suggests that researchers should not criticize the theory that HIV causes AIDS unless they have a better explanation. Students cannot be expected to discover on their own that this criticism has less force in scientific argumentation: hypotheses must stand up to criticism whether or not alternatives exist.

\section{Conclusions and Further Work}

Our ongoing work is motivated by our observations concerning the need for apprenticeship to practices not found in peer groups, and tradeoffs in design of groupware for collaborative learning.

Scaffolding Scientific Argumentation Skills. We are currently pursuing several lines of inquiry into ways to encourage students' existing competencies while scaffolding learning of skills that are not found in everyday argumentation. An automated "advisor" gives advice concerning ways in which an argument can be extended or revised. Such advice is important for exposing students to standards of evaluation that may not exist in their peer groups. It is also expected to stimulate further inquiry when students have reached an impasse. The advisor is discussed further in [18]. Also, we are designing argumentation palettes (the menus of icons, see left side of Figure 1) for everyday and scientific argumentation. The palettes vary in granularity, distinctions available (e.g., whether "justifications" are included as a component type), and relationships emphasized (e.g., theory versus domain). We plan to use these palettes to effect transitions between everyday and scientific argument in a manner fitting students' emerging competencies and readiness to appreciate new distinctions. We also plan to experiment with rhetorical and topical ways of indexing information, the goal being to help students acquire appropriate information-seeking strategies.

Tradeoffs in the Design of Groupware for Learning. The emphasis on stimulating critical discussion complicates the criteria for interface design. Although we design to make it easy to construct diagrammatic representations of the dialectical aspects of science, we also design to stimulate external discourse that need not be recorded in the diagram. We have found that the latter goal can overrule the utility of features we would otherwise provide in support of the former. For example, statements in Belvedere are embedded in shapes that represent their epistemological status. Consequently, students using Belvedere often discuss the epistemological status of a statement before representing it in the diagram. An object can only have one shape at a time; therefore their discussion of the epistemological status cannot be part of the diagram. This might be viewed as a design flaw of the graphical language. However, it may be useful to force a decision prior to entry in the diagram precisely because it stimulates discussion towards making the decision.

Feedback on incoherent or weak constructions provides another example. If Belvedere were a tool for use by expert members of some community of practice, we could assume that users shared standard terminology and practice in argumentation. Incoherent links could be disallowed without explanation, and early notification of incoherent or weak patterns of links might enhance the users' efficiency as argument designers. However, students may have different feedback needs. They do not yet share the semantics of argumentation terminology, and immediate notification of incoherent or weak argument patterns could prevent students from engaging in processes of theory criticism and revision that are encountered in the real world.

Although unresolved, these issues illustrate the difference between local and systemic optimization of groupware for learning. Design to support discourse processes must transcend the representational environment of the software itself, even in software that specifically relies on the utility of on-line representations for discourse. The participants' discourse processes take place in the social environment as well as within the representational and computational resources provided by support software. Thus, the utility of software features should be evaluated in terms of how well they stimulate the right kind of activity in the total human-computer system, not merely in terms of support for local tasks. All of our efforts seek to uncover how productive discourse can be facilitated or stimulated by each feature of the interface and of the task posed to the students, and how we can design the interface and task to support a collaborative transition from everyday to scientific argumentation skills.

\section{Acknowledgments}

This research was conducted while supported by grant MDR-9155715 from the NSF Applications of Advanced Technology program. We also thank Violetta Cavalli-Sforza, John Connelly, Alan Lesgold, 
Arthur Nunes, Massimo Paolucci, and Mike Smith for their collaboration; and Ed Henke, Tom Valco, and Shirley York for access to their students.

\section{References}

1. W. Blattner, R. Gallo, and W. Temin. HIV causes AIDS. Science, 241:515-516, July 1988.

2. J. Braddock and J. McPartland. Education of early adolescents. Review of Research in Education, 19:135-170, 1993.

3. M. W. Browne. Galapagos mystery solved: Fauna evolved on vanished isles. Science Times, The NY Times, January 211992.

4. W. Chase and H. Simon. The Mind's Eye in Chess. In W. Chase (Ed.) Visual Information Processing, pages 251-281. Academic Press, 1973.

5. M. Chi, R. Glaser, and E. Rees. Expertise in problem solving. In R. Sternberg (Ed.) Advances in the Psychology of Human Intelligence, Volume 1, pages 17-76. Erlbaum, 1982.

6. J. Conklin and M. Begeman. gIbis: A hypertext tool for team design deliberation. In Hypertext '87, pages 247-252, Chapel Hill, NC, November 1987.

7. A. deGroot. Perception and memory versus thought: Some old ideas and recent findings. In B. Kleinmuntz (Ed.) Problem Solving, pages 19-50. Wiley, 1966.

8. P. Duesberg. HIV is not the cause of AIDS. Science, 241:514-517, July 1988.

9. G. Fischer, R. McCall, and A. Morch. Janus: Integrating hypertext with a knowledge-based design environment. In Hypertext '89, pages 105-117, Pittsburgh, PA, November 1989.

10. S. Goldman. Computer resources for supporting student conversations about science concepts. http://www.cica.indiana.edu/csc195/outlook/04_ Goldman.html.

11. D. Kuhn. Skills of Argument. Cambridge University Press, 1991.

12. D. Newman, S. V. Goldman, D. Brienne, I. Jackson, and S. Magzamen. Computer mediation of collaborative science investigations. Educational Computing Research, 5:151-166, 1989.

13. D. K. O'Neill and L. M. Gomez. The collaboratory notebook: A distributed knowledge-building environment for project-enhanced learning. In Proceedings of Ed-Media '94, Vancouver, BC, June 1994.

14. M. Scardamalia and C. Bereiter. Higher levels of agency for children in knowledge building: A challenge for the design of new knowledge media. The Journal of the Learning Sciences, 1:37-68, 1991.

15. R. E. Slavin. Cooperative Learning: Theory, Research, and Practice. Prentice-Hall, Englewood Cliffs, NJ, 1990.

16. P. Smolensky, B. Fox, R. King, and C. Lewis. Computer-aided reasoned discourse, or, how to argue with a computer. In R. Guindon (Ed.) Cognitive Science and its Implications for Human-Computer Interaction. Lawrence Erlbaum, 1987.

17. N. Streitz, J. Hannemann, and M. Thuring. From ideas and arguments to hyperdocuments: Traveling through activity spaces. In Hypertext '89, pages 343-364, Pittsburgh, PA, November 1989.

18. D. Suthers, A. Weiner, J. Connelly, and M. Paolucci. Belvedere: Engaging students in critical discussion of science and public policy issues. To appear in AI-Ed 95, the 7th World Conference on Artificial Intelligence in Education.

19. S. E. Toulmin. The uses of argument. Cambridge University Press, 1958.

20. D. Wan and P. M. Johnson. Experiences with CLARE: a Computer-Supported Collaborative Learning Environment. International Journal of Human-Computer Studies, October, 1994.

\section{Authors Addresses}

Dan Suthers and Arlene Weiner. Learning Research and Development Center, 3939 O'Hara Street, University of Pittsburgh, Pittsburgh, PA 15260; +1412-624-\{7036,7056\}; \{suthers, arelene\}+@ pitt.edu; http://www.pitt.edu/\{ $\sim$ suthers, arlene $\}$. 